and diverticulitis were blood volume, mean transit time, permeability, and enlargement of pericolonic lymph nodes (all $P \leq 0.05$ ).

Perfusion measurements are superior to most morphologic criteria to discriminate between colon cancer and diverticulitis; however, a combination of both approaches might be most appropriate in clinical practice, because all values overlapped.

Original article Goh V et al. (2007) Differentiation between diverticulitis and colorectal cancer: quantitative CT perfusion measurements versus morphologic criteria-initial experience. Radiology 242: 456-462

\section{HRT slows fibrosis progression in postmenopausal women with chronic hepatitis C}

Fibrosis progression in women with chronic hepatitis $\mathrm{C}$ can be exacerbated by diabetes, obesity, steatosis and postmenopausal status. Estrogen is thought to inhibit activation of hepatic stellate cells and have an antifibrogenic role; therefore, hormone replacement therapy (HRT) could also have antifibrogenic effects. In this single-center, prospective, crosssectional, French study, Codes and colleagues investigated whether the severity of fibrosis in 251 women with chronic hepatitis C (age range 18.6-72.7 years) was related to postmenopausal status, HRT, or steatosis.

The cohort included 122 postmenopausal women, of whom 65 had received or were receiving HRT (mean duration 2.3 years). All 251 patients underwent a liver biopsy and liver function tests.

In total, 190 women had mild fibrosis and 61 women had moderate or severe fibrosis. On univariate analysis, estimated duration of $\mathrm{HCV}$ infection $>15$ years, BMI $>25 \mathrm{~kg} / \mathrm{m}^{2}$, severe steatosis ( $\geq 30 \%)$, and postmenopausal status were all markedly associated with moderate or severe fibrosis. On multivariate analysis, postmenopausal status was associated with moderate or severe fibrosis; however, the stage of fibrosis was lower in postmenopausal women receiving $\mathrm{HRT}$ than in those not receiving HRT. Steatosis was most frequent and severe in postmenopausal women and those infected with HCV genotype 3 .

The authors conclude that HRT has no deleterious hepatic effects in women with chronic hepatitis C. HRT might have a beneficial effect on fibrosis, owing to the probable antifibrogenic role of estrogens.

Original article Codes L et al. (2007) Liver fibrosis in women with chronic hepatitis $\mathrm{C}$ : evidence for the negative role of the menopause and steatosis and the potential benefit of hormone replacement therapy. Gut 56: 390-395

\section{IFN- $\gamma 1 \mathrm{~b}$ therapy is ineffective in patients with advanced liver fibrosis or cirrhosis}

Preliminary studies indicated that interferon (IFN)- $\gamma 1 \mathrm{~b}$ could potentially inhibit fibrosis of the liver. Pockros and colleagues, therefore, investigated whether this effect occurs in patients with chronic HCV infection and advanced liver fibrosis or cirrhosis.

This prospective, double-blind, randomized, placebo-controlled, multicenter trial evaluated 488 patients (mean age 51.7 years, 69\% male, $70 \%$ white). Most patients (84\%) had cirrhosis (Ishak stage 5 or 6), and the rest had advanced fibrosis (Ishak stage 4). All patients were positive for anti-HCV antibody or HCV RNA. Participants were randomly allocated to receive subcutaneous injections of $100 \mu \mathrm{g}$ IFN- $\gamma 1 \mathrm{~b}(n=169), 200 \mu \mathrm{g}$ IFN- $\gamma 1 \mathrm{~b}$ $(n=157)$, or placebo $(n=162)$ three times per week for 48 weeks. No antiviral medication was administered throughout the study, and the primary end point was a reduction of $\geq 1$ Ishak point on assessment-which included posttreatment liver biopsy-during the 4-week follow-up period.

The proportion of patients with an improved Ishak score did not differ significantly between the groups. Levels of CXC-chemokine ligand 11, a marker of IFN- $\gamma$ activity that may be associated with reduced collagen deposition, was increased at week 24 compared with baseline in both IFN- $\gamma 1 \mathrm{~b}$ treatment groups.

The authors conclude that IFN- $\gamma 1 \mathrm{~b}$ monotherapy for 48 weeks cannot reverse advanced fibrosis or cirrhosis of the liver; however, a longer duration of IFN- $\gamma 1 \mathrm{~b}$ treatment, perhaps in combination with $\mathrm{HCV}$ antiviral therapy or in patients with early-stage fibrosis, might be beneficial.

Original article Pockros PJ et al. (2007) Final results of a double-blind, placebo-controlled trial of the antifibrotic efficacy of interferon- $\gamma 1 \mathrm{~b}$ in chronic hepatitis $\mathrm{C}$ patients with advanced fibrosis or cirrhosis. Hepatology 45: 569-578 